\title{
Tipología de transferencias docentes en la formacion de profesores de ciencias
}

\author{
Transfers tipology in the formation of science teachers \\ María del Carmen Gabriela Flores Talavera
}

Instituto Superior de Investigación y Docencia para el Magisterio (ISIDM).gabi@isidm.commx

\begin{abstract}
Resumen
Resultados de tres estudios. Sujetos: profesores en diferentes niveles de formación. Observación sistemática durante la formación y entrevistas fue la metodología empleada. La transferencia es la relación que hacen los profesores en el espacio y tiempo formativo produciendo generalizadiones que se re-actualizan y manifiestan en acciones de: la práctica docente, otras asignaturas de estudio y en sus proyectos de investigación.
\end{abstract}

Se describe la tipología de transferencias que realizan los docentes hacia otros ámbitos de acción. Los profesores realizaron seis tipos de transferencias de conocimientos para la docencia: cognitivas, retrospectivas, verbales, inducidas, visualizadas y -en-la-acción. La dasificación se basó en dos criterios: el temporal y por su resolución de la tensión entre la teoría y la práctica.

Las transferencias cognitivas son generalizaciones constructivas realizadas en el plano abstracto. Las conexiones que el docente hace se re-actualizan en un nivel teónico; permiten al docente ligar los conocimientos de las diferentes asignaturas para lograr una producción intelectual de calidad y que se pueda evidenciar en su trabajo de investigación. Se sub-clasifican en: inferiores, superiores y concretas. Las transferencias de conocimiento en-la-acción son las que más aportan al desarrollo de competencias profesionales docentes.

\section{Palabras clave}

Transferencias de conocimientos, Formación de docentes, Tipología de transferencias, Profesores de ciencias, Competencias docentes.

\section{Abstract}

This paper shows the results of three studies. The subjects were professors in different teaching training levels. Systematic observation during the formation and interviews to professors was used as the methodology. The transference is the relationship that teachers develop in their formation processes, from which they take their acquired knowledge and abilities to their teaching practices on dassroom settings, other subjects of study and in their investigation projects.

The know-how teaching transfers typology describes what the educators transfer to other fields of action. Teachers under the study made six types of transferences of acquired knowledge for teaching. They are the following: cognitive, retrospective, verbal, induced, visualized and on-theaction. This dassification of six types of transferences was based on how the tension between theory and practice was solved. Cognitive transferences are constructive generalizations made in the abstract plane. The educator connections are re-updated in a theoretical level; they allow the educator to link the knowledge of the different subjects to obtain an intellectual production of quality that can be appreciated in its work of investigation. They are dassified in: inferior, superior 
and concrete. The knowledge transferences in-the-action are those that contribute the most to the development of educational professional competitions.

\section{Introducción}

Con un enfoque cognitivo, el presente es la síntesis y resultado de tres investigaciones: la primera, fue la plataforma de inicio en la que se centró la atención en la actualización de los docentes y sus procesos transferenciales y resolución de la tensión entre teoría-práctica; y dos más, que fueron de profundización y atento seguimiento a los resultados de la primera: Una, se centró en las prácticas y productos de las últimas generaciones de estudiantes de formación inicial y el tipo de transferencias de conocimiento que haćan; y la otra profundizó en las transferencias de conocimiento en el plano abstracto de trabajo en el nivel de posgrado. De esta manera se cubren los tres niveles de la formación de docentes: inicial, actualización y superación.

Eje problematizador

Se parte de la premisa de que existe un reconocido fracaso en los maestros a la hora de transferir los conocimientos y es durante la formación en donde se localiza el problema. Si los profesores se benefician de un proceso formativo; ¿por qué no hay transferencia de conocimientos del ámbito formativo a la práctica docente de manera consistente y sistemática?

Se considera que existe una estructura jerárquica de dominio en las tradiciones de formación docente que implica problemáticas serias en la efectiva transferencia de conocimientos a la práctica en el aula. El discurso de los profesores cambia pero no se evidencian resultados en su práctica; o estos parecen un tanto endebles.

Desde el paradigma cognitivo se estudia al profesor como sujeto cognoscente y considera que la formación permite la generación y transferencia de conocimientos. ¿Cómo aprenden los profesores? ¿Exactamente cuáles transferencias se pueden esperar durante un programa de formación de profesores? ¿Cuál es la permanencia del conocimiento dentro del repertorio pedagógico del profesor que se forma? ¿Cómo se evidencian las transferencias de conocimiento en sus prácticas docentes?

La teoría en la que se sustenta es la tipología de las tansferencias docentes, que permite iniciar con las explicaciones del fenómeno. Las acciones formativas se conciben como "actividades espeć́ficas de producción de conocimientos susceptibles de transferencia a otras situaciones diferentes a la situación de formación" (Barbier, 1993: 26) que genera cambios y transformaciones internos en las personas. Se define transferencia como:

Las relaciones que hacen los profesores en el espacio y tiempo de la formación produciendo generalizaciones en las que se manifiestan las competencias y concepciones de los docentes reactualizándose en la práctica a través de las acciones (Flores, 2003).

Basándose en la definición original de la palabra transferencia que significa "el desplazamiento de una información de un lugar a otro"; son las relaciones conceptuales que el profesor hace durante su formación y las lleva hacia la cotidianidad de práctica docente; se dan cuando el sujeto después de haber encontrado una solución (falsa o correcta) la aplica indiscriminadamente a contenidos ya presentes en los datos pero que antes no habían sido utilizados. Pero aún así el concepto queda limitado. 
Las transferencias docentes se dasificaron conforme a tres aiterios principales: 1) por el nivel en el proceso de elaboración de la transformación; 2) su relación temporal y, por lo tanto, su viabilidad de generalización y aplicabilidad, y 3) la resolución de la tensión entre la teoría y la práctica.

Dentro de todo proceso formativo se dan todos estos tipos de transferencias identificadas, sin embargo se observó en posteriores investigaciones que estas transferencias se dan en dos niveles de desarrollo y por tanto diferencias en la transformación de la práctica: 1) las transferencias plenamente consolidadas, en las que el profesor realiza de manera creativa y constructiva transferencias del ámbito de la formación al ámbito áulico; y se les denominó en-la-acción; y 2) las transferencias en proceso, que pueden ser intentos de generalización de lo aprendido sin lograrlo plenamente, a las que se denominaron: retrospectiva, verbal, inducida y visualizada. Los tipos de transferencias descritos se han podido organizar en un continuum de consolidación de aprendizajes (Véase cuadro $N^{\circ} 2$ ) que hacen referencia a los procesos transferenciales más deseados durante la formación.

Transferencias cognitivas. - Generalizaciones constructivas realizadas en el plano abstracto. No tienen referencia espacial ni temporal ya que las conexiones que el docente hace se re-actualizan en un nivel teórico. Este tipo de transferencias podrían ser consideradas valiosas en formaciones de tipo intelectual (reflexiones filosóficas) que no precisen de transformaciones en la práctica. Se concibe como transferencia cognitiva cuando se utilizan elementos de una asignatura y se generalizan a otra asignatura. En la investigación realizada en posgrado se profundizó en este tipo de transferencias de conocimiento utilizadas en el desarrollo de competencias espećficas para el trabajo intelectual. En el nivel de posgrado, las transferencias cognitivas se multiplicaron auantitativamente, pero se diversificaron cualitativamente, estableciéndose una primera diferenciación:

Cuadro 2. Continuum de consolidación de las transferencias docentes.

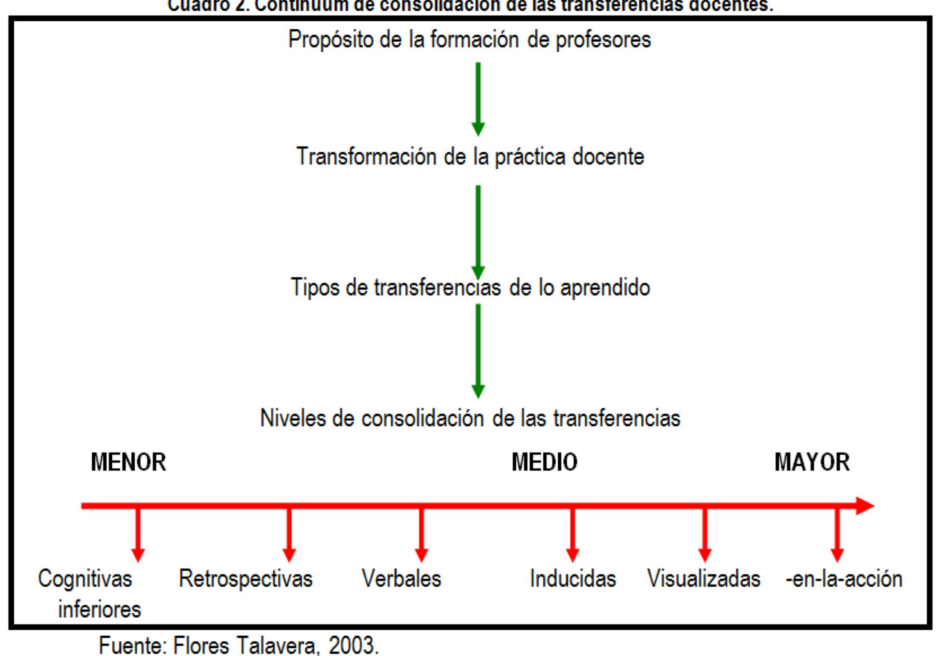

1) Transferencias cognitivas inferiores.- Este tipo de transferencias las realizan los profesores auando reproducen la información sin ningún análisis cuítico previo (repetición memorística); y se caracterizan por ser simples y provenientes de conocimiento inductivo.

2) Transferencias cognitivas superiores.- se considera que el sujeto expresa un aprendizaje por descubrimiento a través de la reflexión y búsqueda de información de un concepto revisado en dase...ampliar la información y por consequente la CREACION de un nuevo conocimiento más complejo. 
3) Iransferencias para dar explicaciones teóricas a lo que sucede en la práctica cotidiana.- y de los fenómenos educativos que está viviendo y así comprender la realidad. Pero son las transferencias de tipo cognitivo; las que permiten al docente ligar los conocimientos de las diferentes asignaturas para lograr una producción intelectual de calidad y que se pueda evidenciar en su trabajo de investigación. Este tipo de transferencias es la que más apoya en estudios de posgrado y al campo del conocimiento teórico educativo.

Transferencias retrospectivas. - Un incidente de esta categońa es cuando el profesor menciona que lo revisado durante el curso o asignatura de la maestría ya lo sabía o lo llevaba a la práctica en el pasado; no intentan comprender la realidad; sino que son más simples. Este tipo de transferencias sirven para comprender los procesos de aprendizaje de los profesores con cierta experiencia en la que tratan de conectar las experiencias anteriores con los nuevos aprendizajes teóricos; esta manera de generalización se califica como negativa porque bloquean un aprendizaje anterior firmemente arraigado en las concepciones docentes, de tal manera que no permiten que el nuevo conocimiento se incorpore. Estas acciones legitimizan su actividad y fundamentan sus pasadas innovaciones empíricas Propician la sedimentación de la práctica actual.

3) Transferencias verbales.- Se caracterizan por generalizar de "algunos" a "todos" los hechos, los conocimientos o las competencias desarrolladas. En este caso no hay creación de nuevas formas o contenidos. Son de tipo impulsivo, a largo plazo; tiene una aplicación remota y probable de los aprendizajes. Son pre-empíricas y poco planeadas, propicia la poca reflexión de las acciones. Las mismas transferencias encontradas pueden ahora explicar dos procesos distintos de relaciones: laterales y verticales, que son transferencias que permiten la elaboración de estructuras cognitivas más complejas.

Las transferencias visualizadas a futuro tienen una aplicación cercana en el aula; son organizadas conforme a las necesidades de los estudiantes y previas a las transferencias-en-laacción de tipo escolar planeada. Son más utilizadas por los futuros profesores que se encuentran en proceso de formación inicial.

Este tipo de transferencias visualizadas se da de manera natural en el profesor que se forma auando hay un proceso firme y sistemático en las acciones del formador. Son las transferencias ideales, en las que se producen generalizaciones constructivas a nivel abstracto (subjetivoindividual), para que después se manifiesten en acciones creativas consolidadas en la acción misma del aula; se considera que se deben generar durante los procesos formativos para que éstos tengan éxito y fructifiquen en la práctica docente posterior.

Transferencias inducidas. - Son propiciadas por el asesor del curso; la reacción observada en los profesores después de que se inducía a una transferencia era la generalización planeada de la habilidad a la práctica del aula. Esta dase de transferencias sirven de andamiaje a las transferencias-en-la-acción y propician seguridad en los profesores porque se retroalimentan a través del diálogo entre los integrantes de la comunidad de aprendizaje. Es importante mencionar que es un recurso utilizado por el asesor con un sentido explíato y con la intención de generar resultados valiosos en la acción de la práctica. Cuando el profesor realiza una transferencia de conocimiento por petición o por sugerencia de una tercera persona también este ejercicio puede ser un andamiaje especial para producir transferencias cognitivas superiores en los estudiantes.

Transferencias en-la-acción. - Cuando el profesor refiere que las competencias desarrolladas durante la formación lo ha llevado al ámbito profesional y lo ha puesto en práctica. Se hacen en el presente, en el aquí y en el ahora, éstas se caracterizan por tener una actuación que las evidencia de manera observable en la cotidianidad de su trabajo docente. Es una generalización de tipo constructiva y creativa y son las ideales para la verdadera transformación de la práctica. 
Las mismas transferencias encontradas pueden ahora explicar dos procesos distintos de relaciones: laterales y verticales, que son transferencias que permiten la elaboración de estructuras cognitivas más complejas (Véase cuadro 3).

Las relaciones transferenciales de tipo lateral se caracterizan por ser inductivas, ya que el profesor aplica indiscriminadamente a otros contenidos o contextos lo aprendido sin una reflexión; se quedan en el primer nivel y sólo se mueven de la teoría a la práctica sin acceder a ámbitos superiores porque están permeadas por los significados que los docentes tienen de la educación y cómo debe de ser.

Los profesores que se quedan en este nivel, deducen que siempre sucederá lo mismo, se limitan a generalizar de "algunos" a "todos" los hechos, los conocimientos o las habilidades desarrolladas. En este caso no hay creación de nuevas formas o contenidos. Las relaciones transferenciales laterales producen transferencias cognitivas inferiores, simples, repetitivas; de poca reflexión y sin complejidad ni abstracción; tal es el caso de las transferencias verbales que se realizan según la utilidad de los conocimientos desde la perspectiva del docente; y las que dan explicaciones a la práctica docente; lo mismo sucederá con las transferencias inducidas fuera de un contexto formativo, ya que su propósito es agradar a la persona que las induce.

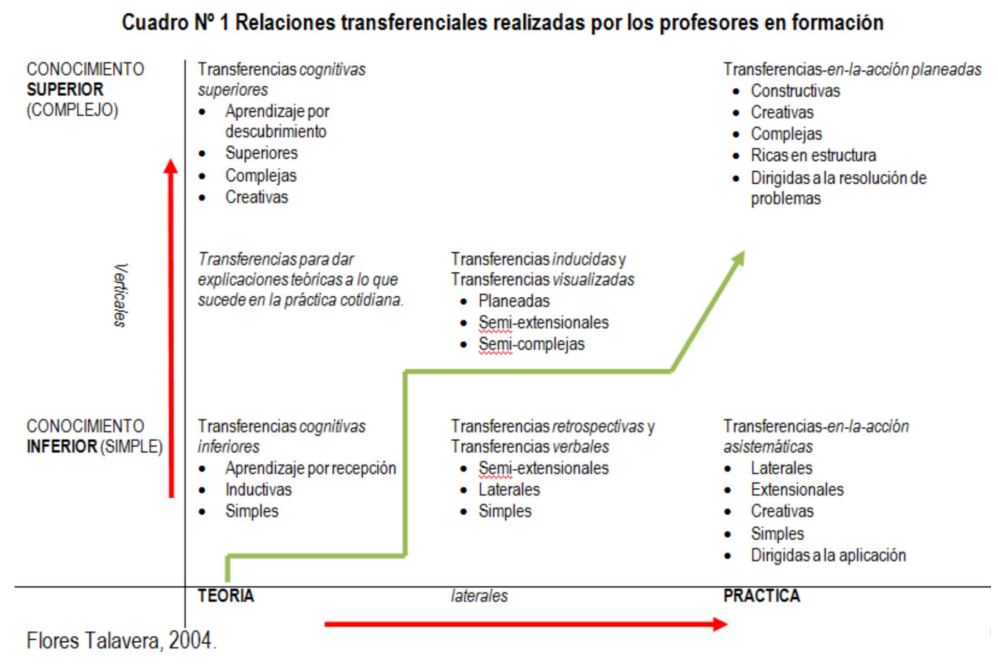

Las relaciones transferenciales de tipo vertical corresponden al grado de transformación de las estructuras cognitivas del profesor. Estas relaciones se caracterizan por la construcción creativa de nuevas formas de pensar y resolver problemas. Es aquí donde se encuentran las tansferencias cognitivas superiores, que se quedan en el ámbito de la teoría pero que permiten a través de las generalizaciones construir y crear conocimiento, nuevo, más rico en estructura, más complejo y superior evidenciado a través de la utilización del conocimiento; por ejemplo en los estudiantes de posgrado los profesores elaboraron su proyecto de investigación.

Los profesores emprenden varios caminos para lograr relaciones transferenciales superiores. Algunos lo hacen lateralmente y luego ascienden hacia la complejidad y la creación. Otros proceden de manera diagonal y siguen la guía de la formación a través de las transferencias inducidas y visualizadas. Otros más se quedan con la información teórica y logran transferencias cognitivas de diferente calidad.

Las variables son diversas, desde las metas parciales que se logran hasta los caminos y formas que los profesores siguen para la transformación de su práctica y por tanto la aplicación y utilización de las competencias docentes recién desarrolladas. Todo ocurre durante el proceso de 
formación, movimiento de estructuras, reflexión y actuación, y que permite lograr las tan complejas transferencias superiores.

\section{Discusión}

Comparación entre la formación inicial, la actualización y el posgrado, para comprender los distintos tipos de transferencias utilizados por los profesores.

Una de la semejanzas que se puede mencionar entre el tipo de transferencias de conocimientos que los profesores realizan en los diferentes niveles de formación (normal, actualización o posgrado) es la motivación. Los significados que los estudiantes tienen impactan profundamente en los tipos de transferencias que realizan. Esto implica las razones por las cuales ingresan a un proceso formativo y las intenciones que tienen para aprender algo.

Otra semejanza es la utilidad del conocimienta. Este proceso en el que el profesor decide por alguna razón que un conocimiento es importante y por tanto digno de ser aprendido es el principio básico de la discriminación y filtro a través de las concepciones del docente para posteriormente hacer transferencias de conocimientos y a partir de este principio la calidad de estas transferencias de conocimientos que van desde las retrospectivas hasta las de en-la-acción.

Pero en este parecido en los tres niveles de formación estudiados, encontramos diferencias sutiles. Por ejemplo, el estudiante de normal encontrará útil un conocimiento cuando le ayuda con el control disciplinario del grupo; con mantenerlos ocupados, divertidos... se resumen a conocimientos de carácter técnico; competencias en el ámbito del saber hacer y repetir estructuras para lograr éxito; sin embargo en posgrado la utilidad del conocimiento radica en la fundamentación teórica para su proyecto de tesis... pero tiene la característica de ser dependiente ya que tiene que ser dicho de manera explíata por el asesor, de otra manera no le encontrarán su utilidad.

Finalmente el profesor en servicio que se forma en cursos o diplomados decide que un conocimiento es útil cuando le "comprueban" que realmente el niño va aprender con esta estrategia y que en la prueba saldrá exitoso. Caso contrario utilizará las transferencias verbales o retrospectivas, con la intención de cuidar su imagen profesional. Las transferencias verbales y enla-acción asistemáticas fueron encontradas en mayor cantidad en los cursos y talleres de actualizadión que en los otros niveles.

Las transferencias cognitivas también son diferentes en cada nivel de formación; por ejemplo, en los estudiantes de posgrado se realizan más en cantidad y pueden ser inferiores aunque existe la tendencia de realizar transferencias cognitivas de carácter superior; no sucede así en la formación inicial, la mayoría de las transferencias cognitivas que realizan los estudiantes normalistas son para pasar los exámenes de las asignaturas. En la actualización las transferencias cognitivas son muy escasas; esto no quiere decir que no se presente solo que las necesidades de conocimiento son diferentes.

Por otra parte las transferencias retrospectivas son más utilizadas por profesores en servicio que están en procesos de formación en talleres cortos, cursos o diplomados... no así se encuentran tanto en profesores que se encuentran en algún posgrado o en la normal.

Las transferencias inducidas se encuentran más frecuentemente en estudiantes de la normal aunque no logran consolidarlas del todo... y en algunos cursos de actualización aryos objetivos tienen la transformación de la práctica. En posgrados teóricos no se encontraron; pero en 
posgrados que tienen como objetivo la transformación de la práctica la probabilidad de que este tipo de transferencias de conocimiento se incremente en cantidad es alta.

\section{Conclusiones y retos para el formador de profesores}

Identificar los tipos de transferencia duraderos permitirá una planeación de la formación con mayor seguridad. Una formación de profesores integral toma en cuenta tres niveles de trabajo: subjetivo-individual; objetivo-individual y objetivo-público; que corresponden a las concepciones previas, las habilidades desarrolladas y su transferencia de conocimientos a otros ámbitos de trabajo del profesor, respectivamente.

En los procesos de formación de profesores que pretendan una verdadera transformación se debe implicar una buena dosis de transferencias planificadas y transferencias-en-la-acción.

Si el proceso formativo tiene la intención y/o desea mejorar o transformar la práctica cotidiana entonces el formador de profesores deberá tener cuidado en el desarrollo de HD que implique un trabajo sobre el sí mismo del profesor, en un ambiente transicional entre la teońa y la práctica con un apoyo constante para propiciar la competencia deseada.

Aún con formaciones docentes cuidadosamente planeados, las concepciones docentes jugarán un papel predominante ya que son los filtros del aprendizaje y de las transferencias a la práctica; por lo que el formador tiene como reto trabajar profundamente en ellas; ya que la práctica docente de un profesor en servicio se ve modificada si y solamente si el proceso de formación le ha impactado en sus concepciones docentes para efectuar la transformación. El reto es continuar con el análisis de los procesos de formación desde el enfoque cognitivo para explicar y comprender la manera en que los profesores aprenden.

Hasta aquí se ha llegado al punto de acercarse a la comprensión del proceso de formación y los procesos transferenciales de conocimientos en los profesores para el desarrollo de competencias; para ello se echó mano de una gran variedad de nociones conceptuales que ayudaron a explicitar el tema que aquí hoy nos ocapa; la formación de docentes considerada como una práctica socialen donde el profesor se somete a un proceso educativo en el que se desarrollan competencias docentes para acompañar el aprendizaje a través de formarse (autoformación); en donde el ser humano trabaja sobre él mismo, sobre sus representaciones y sus conductas impactando su práctica pedagógica y generando transformaciones tanto en el profesional como en las personas con las que trabaja.

Asimismo, es conveniente recordar la focalización de las actitudes como eje de la formación de docentes, principalmente en un modelo integrador; la identificación de sus necesidades para optimizar la formación y sobre todo sus niveles de transferencia duraderos. Es pertinente concebir dentro de la formación docente estrategias para propiciar no sólo el manejo de contenidos, habilidad que tempranamente es adquirida en los aursos formativos, sino el cambio estructural y actitudinal en los docentes.

\section{Bibliografía}

Ausubel, D., Novak, J., \& Hanesian, H. (1983). Psicología Educativa. México: Trillas.

Barbier, J.M (1993). La evaluación en los procesos de formación. Barcelona: Paidos. 
Ducoing Watty, P. (2000). Sobre la formación y la formación de profesores. En E. Matute, \& R. M. Romo Beltrán, Diversas perspectivas sobre la formación docente. Guadalajara, México: Universidad de Guadalajara.

Ferry, G. (1991). El trayecto de la formación. Los enseñantes entre la teoría y la práctica. Barcelona: Paidos.

Flores Talavera, G. (2004). De la formación a la práctica docente. Un estudio de los procesos de transferencia de los profesores. Revista Latinoamericana de estudios educativos, XXXVV(3), 37-68.

Flores Talavera, G. (2003). Formación de Educadores Holistas, el desarrollo de las habilidades docente y sus procesos de transferencia. Guadalajara: Tesis Doctoral. ULSAG.

Flores Talavera, G. (2006) Transferencias cognitivas en estudiantes de posgrada. Informe de investigación Guadalajara: ISIDM. (Mecanograma).

Flores Talavera, G. (2007). Transferencias de conocimientos docentes a la práctica profesional. En AAV, Procesos formativos en el campo de la educación: una experiencia institucional (págs. 6797). Guadalajara: ISIDM-CIPS-ULSAG-UPN.

García Alvarez, J. (1993). La formación permanente del profesorado: más allá de la reforma. . Madrid: Escuela Española.

Herraiz, M. (1999). Formación de Formadores. México. Limusa.

Herraiz, M. (2000). Formación de Formadores. Manual Didáctico. México. Limusa.

Putnam, R. T., \& Borko, H. (2000). El aprendizaje del profesor: implicaciones de las nuevas perspectivas de la cognición. En B. J. Biddle, T. L Good, \& I. F. Goodson, La enseñanza de los profesores I (Vol. I, págs. 219-309). Barcelona, España: Paidos. 